\title{
Yeasts as models in evolutionary biology Zhenglong $\mathrm{Gu}^{*}$ and Stephen Oliver ${ }^{\dagger}$
}

\author{
Addresses: *Division of Nutritional Sciences, Cornell University, Ithaca, NY 14853, USA. "Department of Biochemistry, University of \\ Cambridge, Sanger Building, Tennis Court Road, Cambridge CB2 1GA, UK.
}

Correspondence: Zhenglong Gu. Email: zg27@cornell.edu; Stephen Oliver. Email: sgo24@cam.ac.uk

Published: 2 March 2009

Genome Biology 2009, 10:304 (doi:10.1 I86/gb-2009-10-3-304)

The electronic version of this article is the complete one and can be found online at http://genomebiology.com/2009//0/3/304

(C) 2009 BioMed Central Ltd

A report of the workshop 'Evolutionary and Environmental Genomics of Yeasts', Heidelberg, Germany, I-5 October 2008.

Around 100 scientists gathered at Heidelberg recently for a workshop exploring the burgeoning use of yeasts in studies of evolution and ecology. Yeasts as model systems permit an ideal marriage between theory and experiment, and the meeting concentrated on four core issues in evolutionary biology: the architecture of the genome and its evolution; the ecological and genetic structure of natural populations; the mechanisms of selection that lead to adaptation, and their study in the laboratory; and the evolution of sex and mating systems. Here, we report a few of the highlights.

\section{Genome structure and evolutionary process}

The genome sequences of many yeast species and strains are now available, providing a playground for evolutionary biologists to pursue comparative genomics. Bernard Dujon (Institut Pasteur, Paris, France) presented the work of the Génolevures consortium on the genome evolution of hemiascomycetous yeasts, focusing on the evolution of novel genes. The common ancestor of the Saccharomyces 'sensu stricto' species complex (Saccharomyces species that can mate to produce viable hybrids) underwent a whole-genome duplication (WGD) around 100 million years ago, and as Dujon described, this event had a profound effect on the genome structure of the affected species and led to the emergence of many genes with novel functions. In this regard, Jed Dean (Stanford University, USA) discussed their work showing that members of paralogous pairs of genes created by WGD still exhibit a significant amount of functional redundancy, which is thus a potential source of further genetic innovation.
Aerobic fermentation emerged in different yeast species at the end of the Cretaceous period when fruit sugars became increasingly available. Yitzhak Pilpel (Weizmann Institute, Rehovot, Israel) has found that genes needed for oxidative respiration or fermentation have distinct translational efficiencies in obligate aerobic or facultative species, respectively. He used a measure of translation efficiency to show that genes and functional modules have correlated patterns of translational efficiency across species, and sometimes even emerged independently in markedly different evolutionary lineages (for example, $S$. cerevisiae and Schizosaccharomyces pombe).

\section{Population structure and genetic mapping}

One important issue for population genetics is to infer population history and population structure from current patterns of genetic variation. 'Population genomics' has now emerged as a new field as complete genome sequences of an increasing number of yeast strains become available. The population dynamics of members of the Saccharomyces 'sensu' stricto' group were addressed by Vassiliki Koufopanou (Imperial College, London, UK), who presented the results of sequencing chromosomes III from 20 isolates of $S$. paradoxus. Chromosome III determines the sex of yeast cells and her analysis indicated that $S$. paradoxus living on oak trees may go through a sexual cycle approximately once in every 1,000 asexual generations. This is our first intimation of how frequently yeast perform the sexual act in at least one of their natural environments.

Ed Louis (University of Nottingham, UK) presented the results of sequencing the genomes of around 70 natural isolates of $S$. cerevisiae and S. paradoxus from various geographical locations and ecological environments. Population genetics analysis indicated that the $S$. paradoxus strains divided into three distinct populations plus a single 
Hawaiian isolate that differed from all the rest. In contrast, most of the polymorphisms in the $S$. cerevisiae samples were shared, indicating extensive recombination between lineages in this species. Various evolutionary forces, such as purifying selection and adaptive evolution, were detected in both coding and regulatory sequences by analyzing the spectrum of allele frequency distributions. Louis also mentioned that phenotypic analyses for some 200 growth traits were being carried out for all sequenced isolates in the hope of identifying associations between genotype and phenotype.

A better understanding of yeast population structure should lead to improved methods for association mapping. The high meiotic recombination rates in yeast also make it a good system for rapid mapping of the genetic determinants of phenotypic differences between strains. Lars Steinmetz (European Molecular Biology Laboratory (EMBL), Heidelberg, Germany) reported on the mapping of genes that contribute to the high-temperature-growth ( $\mathrm{Htg}$ ) phenotype of several pathogenic S. cerevisiae strains. Surprisingly, besides identifying a major locus in the pathogenic Htgpositive parent, he and colleagues also identified a gene in the non-pathogenic parent that made a significant contribution to the Htg phenotype of the hybrid. All involved in quantitative trait locus mapping, whether in yeasts, plants or humans, would do well to pay attention to such phenomena. Steinmetz also presented the results of a study in which whole-genome tiling arrays were used to genotype approximately 200 tetrads from a cross between two $S$. cerevisiae strains. This dense genetic map will open the door to new studies of the impact of meiotic recombination on yeast evolution and the panel of tetrads analyzed will provide a superb platform for identifying genetic loci underlying yeast pathogenesis and related phenotypes.

\section{Evolutionary mechanisms}

Mutation is the ultimate source of evolution; it is thus necessary to determine how often mutations occur in nature. Using forward mutations to canavanine resistance at the CAN1 locus, Akira Shinohara (University of Osaka, Japan) showed that mutation frequencies in meiosis are five- to tenfold higher than in mitosis. Andrew Murray (Harvard University, Cambridge, USA) discussed genome-wide variation in mutation rates. By placing URA3 as a marker gene at multiple sites along $S$. cerevisiae chromosome VI, he and his colleagues found that the variation in the frequency of mitotic mutation rate at these different sites correlated with the timing of their DNA replication.

Other than the mutation rate, what else determines the rate of evolution? Claus Wilke (University of Texas, Austin, USA) showed that mistakes in mRNA translation that result in protein misfolding are major forces shaping the evolution of coding sequences. Highly expressed genes, in particular, evolve their coding sequences so as to maximize translational fidelity. This explanation of the covariance of coding sequences, codon usage, and mRNA levels is likely to hold true for all branches of life. The importance of protein folding was also emphasized by Daniel Jarosz (Whitehead Institute for Biomedical Research, Cambridge, USA), who showed that the chaperone protein Hsp9o influences the expression of new phenotypes during evolution. Repeated selection can allow the new phenotypes to become Hsp9oindependent, indicating that Hsp9o is important for the assimilation of these new phenotypes into the organism's repertoire, but not necessarily for their continued stable expression.

Genes do not evolve in isolation. Rather, their course of evolution is the product of their interaction with other genes and with the environment. Ryszard Korona (Jagiellonian University, Krakow, Poland) has compared the growth of both single and double deletants of $S$. cerevisiae in benign and stress conditions. The comparison suggested an average alleviating effect of interactions between stressful environments and gene deletions, and among the gene deletions themselves. Paul Magwene (Duke University, Durham, USA) discussed an interesting case of systematic pleiotropy between sporulation and pseudohyphal growth in yeast. A survey of the two phenotypes in different wild and laboratory yeast isolates indicates that a phenotypic trade-off led to significant antagonistic pleiotropy during evolution.

Yeast is ideal for laboratory evolution experiments because of its short generation time and the separate haploid and diploid phases of its life cycle. Maitreya Dunham (University of Washington, Seattle, USA) has identified mutations underlying the organism's adaptation to various nutrientlimiting conditions. Only a small number of mutations were associated with each condition, indicating that adaptation can occur in very few steps - at least, in laboratory conditions under strong selection. Rong Li (Stowers Institute, Kansas City, USA) discussed an interesting case of laboratory adaptation of myo1 deletants that overcame the severe growth defects that are the consequence of the loss of this gene. The results indicated that polyploidization and aneuploidy are strongly correlated with favorable evolutionary outcomes, an observation echoed by Dunham's study. A theoretical population genetics model presented by Sarah Otto (University of British Columbia, Vancouver, Canada) drew attention to the importance of polyploidy in masking the effects of deleterious mutations, while demonstrating that most populations converged on the diploid state over time, with tetraploids being particularly disfavored.

\section{Evolution of mating and speciation}

The theory of sexual selection predicts that if an allele confers an advantage in the competition for mates, it will spread through a population. Duncan Greig (University College London, UK) has found that, under high sexual 
selection, yeast strains that produce increased levels of the $\alpha$ mating pheromone are preferred during mating and invaded a population of weak signalers - a phenomenon directly predicted by the sexual selection theory. The downside to heightened pheromone production is that it may impose a selective cost during vegetative growth. The separation of sexual and asexual growth in the yeast life cycle permitted a direct test of this idea by Greg Lang (Princeton University, USA), who reported a trade-off between growth and mating efficiency in yeast, indicating that loss of genes involved in mating-pheromone production could be adaptive for the organism during vegetative growth, an event that is frequently observed in yeast during experimental evolution.

In regard to speciation, Jun-Yi Leu (Academia Sinica, Taiwan) discussed the nuclear-mitochondrial genome incompatibility that leads to hybrid sterility in crosses between $S$. bayanus and $S$. cerevisiae, and showed that the nuclear-encoded mitochondrial AEP2 gene on chromosome XIII of $S$. bayanus was incompatible with $S$. cerevisiae mitochondria. In a similar system, Oliver Zill (University of California, Berkeley, USA) showed that the S. cerevisiae SIR4 gene cannot silence the $S$. bayanus $H M$ loci, another possible reason for the incompatibility between the two species. Interestingly, Zill showed that SIR4 might be under strong positive selection in the Saccharomyces 'sensu stricto' clade.

Geraldine Butler (University College Dublin, Ireland) discussed results from sequencing a group of Candida species that translate CTG as serine instead of leucine. Members of this clade differ greatly in their ability to undergo sexual reproduction - some have perfect sexual cycles, others show cryptic sexuality, and others are completely sterile. Butler has found that these differences relate to the loss of genes involved in the pathway leading to meiosis, with even the fully sexual species showing a mechanism of meiosis more similar to that of Drosophila than to that of Saccharomyces. Whether such gene loss was the cause of reproductive isolation that produced the different species, or whether it occurred subsequent to speciation, is yet to be resolved.

The mechanism of post-zygotic isolation in speciation was also studied at the protein level by Daniela Delneri (University of Manchester, UK), who investigated the ability of hybrids between different Saccharomyces 'sensu stricto' species to assemble protein complexes containing components from both parents. Examples of chimerism were found for all three protein complexes studied, but the ability to form chimeras did not correlate with the degree of sequence divergence between species.

\section{New frontiers in evolutionary biology}

On the systems biology front, one of us (SO) discussed the results of competition experiments between heterozygous yeast deletants grown in both nutrient-limiting (chemostat) and nutrient-sufficient (turbidostat) cultures. Both haploinsufficient (heterozygotes that grow more slowly than the wild type) and haploproficient (heterozygotes that grow faster than wild type) were found in all selective environments. Haploproficiency was revealed less often in turbidostat culture than in chemostats, but its very existence under nutrient-unconstrained conditions suggests that, over evolutionary time, yeast has sacrificed increased growth rate to improved fidelity of reproduction.

Chiraj Dalal (Caltech, Pasadena, USA) reported a novel mode of regulating gene transcription. The Crz1 zinc-finger transcription factor was shown to exhibit bursts of nuclear localization upon exposure of the cells to calcium. Interestingly, different calcium concentrations controlled the frequency, but not the duration, of these bursts. In this way, frequency-modulated control can coordinate expression from multiple promoters having different threshold functions. Chris Landry (University of Montreal, Montreal, Canada) presented a novel method, the protein fragment complementation assay (PCA), to carry out genome-wide screens for protein-protein interactions. The method identified many novel in vivo interactions, revealing a previously unknown domain of the yeast protein interactome and winning the best poster award for Landry.

This meeting demonstrated that our deep understanding of yeasts and the advanced tools available for their genetic manipulation could mean that these organisms will become as important for research in evolutionary biology as they have been in cellular and molecular biology. Evolutionary biologists will have to be prepared to exploit the potential ability to sequence a yeast genome for a few hundred dollars, while yeast researchers will have to adopt an increasingly evolutionary perspective in their understanding of these organisms. As Theodosius Dobzhansky put it: "Nothing in biology makes sense except in the light of evolution". Yeasts are no exception. 